\title{
COVID-19: biologic and immunosuppressive therapy in gastroenterology and hepatology
}

Markus F. Neurath ${ }^{1,2}$

Abstract | The coronavirus disease 2019 (COVID-19) pandemic is an ongoing global health crisis causing major challenges for clinical care in patients with gastrointestinal diseases. Although triggering of anti-viral immune responses is essential for clearance of infection, some patients have severe lung inflammation and multiorgan failure due to marked immune cell dysregulation and cytokine storm syndrome. Importantly, the activation of cytotoxic follicular helper T cells and a reduction of regulatory $T$ cells have a crucial, negative prognostic role. These findings lead to the question of whether immunosuppressive and biologic therapies for gastrointestinal diseases affect the incidence or prognosis of COVID-19 and, thus, whether they should be adjusted to prevent or affect the course of the disease. In this Review, data on the use of such therapies are discussed with a primary focus on inflammatory bowel disease, autoimmune hepatitis and liver transplantation. In particular, the roles of corticosteroids, classic immunosuppressive agents (such as thiopurines and mycophenolate mofetil), small molecules (such as Janus kinase (JAK) inhibitors), and biologic agents (such as tumour necrosis factor (TNF) blockers, vedolizumab and ustekinumab) are reviewed. Finally, the use of severe acute respiratory syndrome coronavirus 2 (SARS-CoV-2) vaccines for the prevention of infection in patients with gastrointestinal diseases and concomitant immunosuppressive or biologic therapy will be discussed.

The coronavirus disease 2019 (COVID-19) pandemic is a serious global health threat caused by severe acute respiratory syndrome coronavirus 2 (SARS-CoV-2), with more than 179 million cases and 3.8 million confirmed deaths worldwide as of 24 June 2021 (see Related links). Initial studies demonstrated active infection of the upper and lower airways with SARS-CoV-2 $\left(\mathrm{REFS}^{1-9}\right)$. Although many patients have mild flu-like symptoms, some patients develop severe disease frequently associated with the clinical manifestation of pneumonia. Pulmonary infection can be followed by acute respiratory distress syndrome and multiorgan failure, thereby contributing to the marked mortality in patients with SARS-CoV-2 infection ${ }^{2,10,11}$. Mortality is substantially related to cofactors such as age, environmental factors such as smoking and comorbidities such as diabetes, hypertension, or lung and heart diseases ${ }^{12}$.

Detailed studies of SARS-CoV-2 by sequencing analyses revealed a close relationship to two previously known bat-derived SARS-like coronaviruses ${ }^{13}$. Genetic studies indicate that SARS-CoV-2 took advantage of specific mutations and recombination events in the membrane, envelope, nucleocapsid and spike glycoprotein regions to become a novel infectious agent and to adapt to a human host ${ }^{8}$.

The surface spike glycoprotein (S protein) of SARS-CoV-2 enters human cells via a specific surface receptor, angiotensin-converting enzyme $2(\mathrm{ACE} 2)^{14-18}$. This monocarboxypeptidase regulates the cleavage of several peptides within the renin-angiotensin system and is involved in regulation of the intestinal amino acid transporter B0AT1, whose activity controls tryptophan homeostasis. Moreover, ACE2 is involved in the expression of antimicrobial peptides and the ecology of the gut microbiota ${ }^{17}$. A high expression of ACE2 is noted in type 2 pneumocytes in the lung ${ }^{15,16,19}$, which explains the preference of SARS-CoV-2 infection for the airways. In addition to ACE2, the cell surface-associated transmembrane serine protease 2 (TMPRSS2) controls cleavage and activation of the $\mathrm{S}$ protein and thereby regulates viral uptake ${ }^{9,19}$. Upon cell entry of the virus via interactions with ACE2 and TMPRSS2, the RNA of SARS-CoV-2 reaches the cytoplasm of infected cells, resulting in the generation of accessory and structural proteins. Subsequently, viral particle buds and virion-containing vesicles that are released from 


\section{Key points \\ - The coronavirus disease 2019 (COVID-19) pandemic is a global health threat caused by severe acute respiratory syndrome coronavirus 2 (SARS-CoV-2), which binds to cellular angiotensin-converting enzyme 2 (ACE2) and cell surface-associated transmembrane serine protease 2 (TMPRSS2). \\ - As ACE2 and TMPRSS2 are expressed on the surface of several cell subsets in the gastrointestinal tract (such as intestinal epithelial cells), COVID-19 frequently causes gastrointestinal manifestations of disease. \\ - Patients with gastrointestinal disorders, such as inflammatory bowel disease (IBD), and liver transplant recipients should be encouraged to follow strict hygiene and social distancing measures. \\ - In patients with IBD, high-dose corticosteroid treatment before SARS-CoV-2 infection has been identified as a risk factor for aggravated outcomes, suggesting that corticosteroid therapy should be reduced if possible. \\ - Although systemic corticosteroids are recommended for the treatment of patients with severe COVID-19 in intensive care units, these drugs might augment mortality in non-severe COVID-19 cases. \\ - Immunosuppression in IBD and liver transplant recipients appears not to induce aggravated outcomes and might even exert a protective effect against severe COVID-19; patients should consider vaccination whenever available.}

infected cells and enable the dissemination of infection are generated ${ }^{20}$.

In this Review, I discuss the role of gastrointestinal infection in COVID-19 and address the question of whether immunosuppressive and biologic therapies for gastrointestinal diseases affect the incidence or prognosis of COVID-19 infection. In particular, therapies in the context of inflammatory bowel disease (IBD), autoimmune hepatitis and liver transplantation will be discussed.

\section{Gastrointestinal infection in COVID-19}

Although initial data suggested rare involvement of the gastrointestinal tract in COVID-19, more recent findings have highlighted the fact that gastrointestinal symptoms, such as abdominal pain, loss of appetite and diarrhoea, are present in 30-70\% of patients ${ }^{21-24}$. A particularly high prevalence of gastrointestinal symptoms is noted in patients with cancer and COVID-19 in general. For instance, in one study, 36 of 395 hospitalized patients with COVID-19 had an active malignancy ${ }^{25}$. Interestingly, gastrointestinal symptoms might be caused by direct infection of the intestinal tract, as tongue epithelial cells, pharyngeal cells, stomach cells and epithelial cells of the small and large intestine all express ACE2 (REFS ${ }^{26,27}$ ). The highest expression levels of ACE2 with apical co-expression of TMPRSS2 are detected in human intestinal epithelial cells of the terminal ileum ${ }^{19,27}$ (FIG. 1). Infection of human intestinal epithelial cells was confirmed by studies in organoids, whereby SARS-CoV-2 infection led to the induction of a viral response programme and the production of infectious viral particles ${ }^{28}$. However, it should be noted that SARS-CoV-2 mRNA rather than live viruses have been detected in stool of patients with COVID-19 (REF. ${ }^{5}$ ), suggesting that gastrointestinal infection might be self-limiting.

In addition, SARS-CoV-2 infection might directly affect the liver and bile ducts as cholangiocytes and, to a lesser extent, hepatocytes express ACE2 and TMPRSS2.
Consistently, up to $60 \%$ of patients with COVID-19 have elevated liver enzymes and exhibit abnormal liver function at clinical presentation ${ }^{29-31}$. Liver enzymes (alanine aminotransferase, aspartate aminotransferase) are particularly elevated in patients with severe COVID-19, although this observation might be attributable to systemic inflammation, medication, hypoperfusion, and comorbidities or cofactors (such as alcohol consumption).

\section{Immune cell changes in COVID-19}

An activation of immune cells has been noted in COVID-19, which might lead to effective anti-viral immune responses and clearance of viral infection ${ }^{32,33}$ (FIG. 1). In particular, cells of the adaptive immune system, such as B and T cells, have been suggested to have a key role in driving anti-viral immunity. Antigenspecific antibody production by human B cells as well as $\mathrm{T}$ cell-specific memory responses can be induced by SARS-CoV-2 infection. In severe cases, SARS-CoV-2 infection leads to death of activated immune cells. Accordingly, lower lymphocyte counts as well as lower levels of monocytes, eosinophils, basophils and mucosa-associated invariant $\mathrm{T}$ cells are detected in the peripheral blood of these patients $\mathrm{s}^{34,35}$. Moreover, reduced numbers of blood $\mathrm{CD}^{+}$and $\mathrm{CD}^{+} \mathrm{T}$ cells are found, indicating suppression of anti-viral immune cell function in the host in severe COVID-19 that results in unrestrained viral dissemination and organ injury ${ }^{36}$. By contrast, neutrophilia are present in patients with severe COVID-19 and activated neutrophils have been suggested to contribute to fatal outcomes by the production of pro-inflammatory cytokines and vascular occlusions via neutrophil extracellular traps ${ }^{37,38}$.

Although the percentage of naive helper T cells $\left(\mathrm{T}_{\mathrm{H}}\right.$ cells) is increased in patients with COVID-19, memory helper $\mathrm{T}$ cell numbers are decreased ${ }^{36,39}$. Additionally, patients with severe COVID-19 have reduced numbers of regulatory $\mathrm{T}$ cells $\left(\mathrm{T}_{\text {reg }} \text { cells }\right)^{34}$, a cell subset that suppresses pro-inflammatory immune responses via cell-cell contacts and produces anti-inflammatory cytokines such as IL-10 and TGF $\beta$. This reduction in $\mathrm{T}_{\text {reg }}$ cell numbers has been suggested to contribute to hyperinflammation and the cytokine storm syndrome in patients in intensive care with higher plasma levels of various cytokines, including IL-2, IL-7, IL-10, granulocyte colony-stimulating factor (G-CSF), CXCL10, CCL2, CCL3 and tumour necrosis factor (TNF) ${ }^{32,33}$. This syndrome might trigger fatal outcomes via multiorgan failure in COVID-19. Although COVID-19 is usually less severe in children and infants ${ }^{40}$, a severe systemic inflammatory reaction, known as paediatric inflammatory multisystem syndrome or multisystem inflammatory syndrome in children, might occur in these patients and is also associated with elevated serum levels of various pro-inflammatory cytokines ${ }^{41}$.

\section{$T$ and $B$ cells and cytokine responses}

Various studies analysed $\mathrm{T}$ and $\mathrm{B}$ cell activation as well as cytokine responses in COVID-19. SARS-CoV-2 causes broadly directed, diverse and functionally replete memory $\mathrm{CD}^{+}$and $\mathrm{CD}^{+} \mathrm{T}$ cell responses ${ }^{42,43}$. 
Convalescent-phase SARS-CoV-2-specific T cells can be identified in antibody-seronegative exposed family members and convalescent individuals with a history of asymptomatic or mild COVID-19. CD4 ${ }^{+} \mathrm{T}$ cell responses to spike proteins are correlated with the magnitude of the anti-SARS-CoV-2 B cell response as indicated by IgG and IgA antibody titres ${ }^{44}$. In addition, the expansion of highly cytotoxic effector $\mathrm{T}$ cell subsets, such as $\mathrm{CD} 4^{+}$ effector-granulysin, $\mathrm{CD}^{+}$effector-granulysin and natural killer T CD160 cells, is observed during recovery ${ }^{45}$.

Although higher proportions of protective SARSCoV-2-specific $\mathrm{CD}^{+} \mathrm{T}$ cells are observed in mild COVID-19 (REFS ${ }^{46,47}$ ), the breadth and magnitude of $\mathrm{T}$ cell responses are significantly higher in severe cases. Moreover, in severe COVID-19, T cells show signs of senescence, a state in which cells can no longer divide, and exhaustion, a state of $\mathrm{T}$ cell dysfunction, suggesting that effective memory $\mathrm{T}$ cell responses are markedly altered in severe COVID-19 and impair optimal control of infection ${ }^{10}$. Augmented IFNa cytokine responses play a predominant role in COVID-19 (REF. ${ }^{45}$ ). Additional findings revealed a marked increase in the number of $\mathrm{T}_{\mathrm{H}} 17$ cells and $\mathrm{T}_{\mathrm{H}} 17$-related cytokines and transcription factors (specifically, IL-17, IL-23, RAR-related orphan receptor- $\gamma(\mathrm{ROR} \gamma \mathrm{t}))$ in the blood of patients with COVID-19 compared with controls ${ }^{34}$. Although neutrophils suppress $\mathrm{T}_{\mathrm{H}} 1$ cytokine responses, $\mathrm{T}_{\mathrm{H}} 17$ cell development in COVID-19 is triggered by neutrophils and is correlated with poor clinical outcomes ${ }^{48}$. In agreement with a crucial role for $\mathrm{T}_{\mathrm{H}} 17$ cells, genetic studies and Mendelian randomization indicated a causal link between a high expression of tyrosine kinase 2 (TYK2; the Janus kinase that mediates IL-23 signalling) and life-threatening disease ${ }^{49}$. In contrast to the increase in $\mathrm{T}_{\mathrm{H}} 17$ cells, patients show a remarkable reduction in the frequency of $\mathrm{T}_{\text {reg }}$ cell-related proteins such as forkhead box protein P3 and cytokines (TGF $\beta$ and IL-10) (2. $^{34}$. Finally, $\mathrm{T}_{\mathrm{H}} 1$ rather than $\mathrm{T}_{\mathrm{H}} 17$ responses are associated with anti-viral immune responses during SARS-CoV-2 vaccination in a vaccine trial with BNT162b1, a lipid nanoparticle-formulated nucleoside-modified mRNA that encodes the receptor binding domain of the SARS-CoV-2 spike protein ${ }^{50}$.

Single-cell transcriptomic analysis of viral antigenreactive $\mathrm{CD}^{+} \mathrm{T}$ cells showed increased proportions of cytotoxic $\mathrm{T}$ follicular helper cells and cytotoxic $\mathrm{CD} 4^{+}$ cytotoxic T lymphocytes responding to SARS-CoV-2 in patients with severe COVID-19 compared with non-hospitalized patients. In the former group, a strong cytotoxic $\mathrm{T}$ follicular helper cell response was observed early in the illness, which correlated negatively with antibody levels against the spike protein ${ }^{51}$.

Collectively, these findings underline the complex immune dysregulation in COVID-19. Although severe

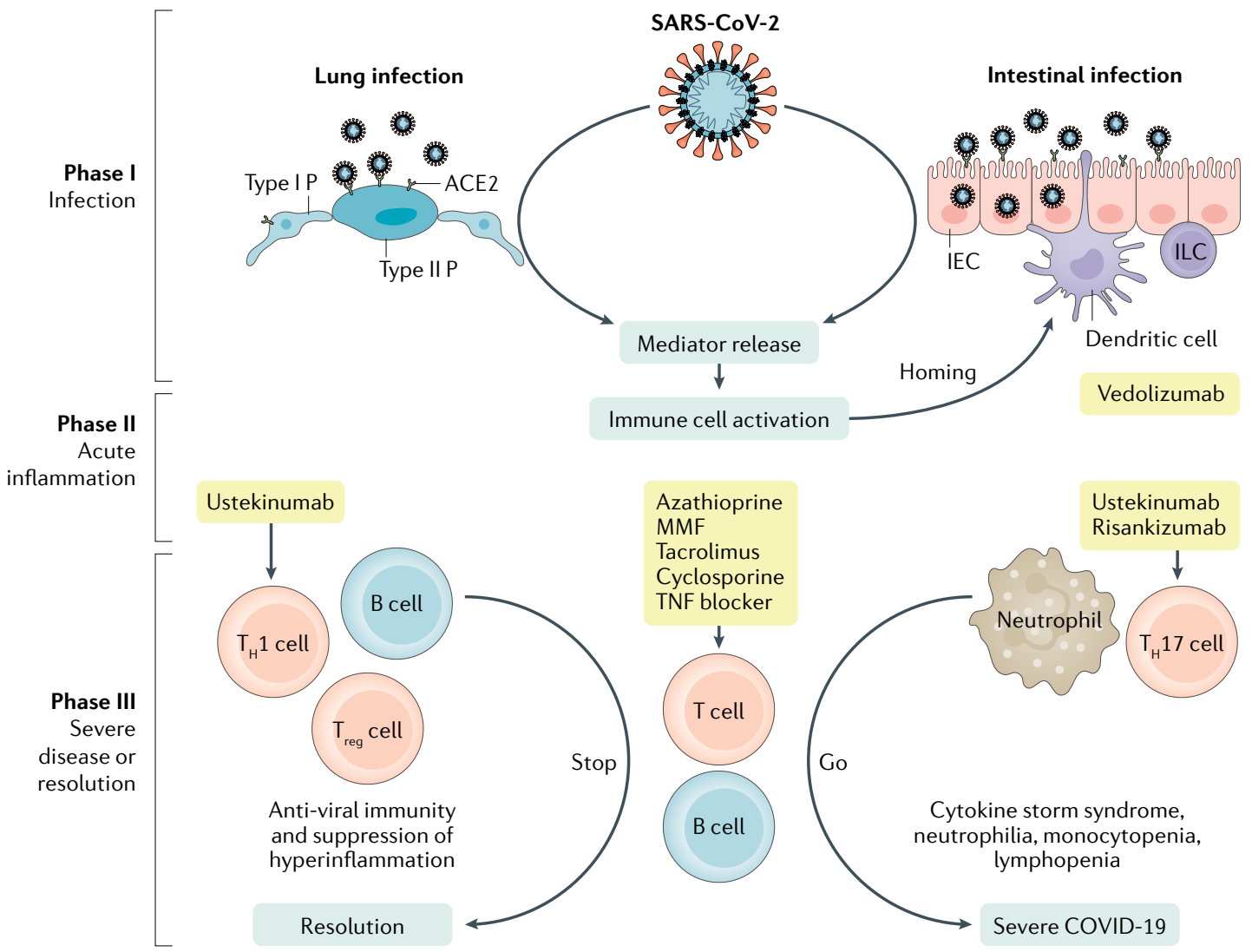

Fig. 1 | Immune cell activation in COVID-19 and potential intervention checkpoints of biologic and immunosuppressive therapies in gastrointestinal disease. Intervention strategies are highlighted in yellow. ACE2, angiotensin-converting enzyme 2; COVID-19, coronavirus disease 2019; IEC, intestinal epithelial cell; ILC, innate lymphoid cell; MMF, mycophenolate mofetil; SARS-CoV-2, severe acute respiratory syndrome coronavirus 2 ; $\mathrm{T}_{\mathrm{H}} 1$, T helper 1 ; $\mathrm{T}_{\text {reg, }}$, regulatory T; Type I P, type 1 pneumocyte; Type II P, type II pneumocyte. Adapted with permission from REF. ${ }^{24}, \mathrm{BMJ}$. 
COVID-19 is associated with marked systemic immunosuppression, local infection in tissues such as the lung is characterized by the activation of immune cells and hyperinflammation associated with a cytokine storm syndrome ${ }^{33,52}$. In the context of gastrointestinal disorders, it is therefore of utmost importance to understand the effect of immunosuppressive and biologic therapies on the clinical course of COVID-19. For instance, are patients with IBD and liver transplant recipients at higher risk of severe COVID-19 due to drug therapy (TABLE 1)? In particular, what is the role of classic immunosuppressive drugs, such as corticosteroids, in this context and should immunosuppressants or biologic agents be stopped or reduced in COVID-19? In the following sections, the latest studies on these agents in patients with gastrointestinal disorders are discussed.

When subsequently discussing implications for therapy, it should be noted that we are still missing interventional controlled studies and that recommendations are mainly based on case series and observational analyses. Moreover, patients on biologic or immunosuppressive agents are often closely followed by physicians and might undergo testing for COVID-19 when symptoms are milder than in patients on other medications or the general population. Thus, there might be detection bias, as mild COVID-19 is more often diagnosed in patients on cytokine inhibitors ${ }^{53}$, and this aspect might lead to the overrepresentation of mild cases.

\section{COVID-19 and IBD pathogenesis}

In patients with Crohn's disease, a high expression of ACE2 and TMPRSS2 in ileal and colonic epithelial cells is observed ${ }^{26,27}$. However, ACE2 (mRNA and protein levels) but not TMPRSS2 expression is substantially lower in inflamed ilea compared with uninflamed control patients and is negatively correlated with inflammation markers such as S100A8 $\left(\right.$ REF. $\left.^{27}\right)$. ACE2 is particularly reduced in patients who subsequently develop severe disease $^{54}$. By contrast, expression levels of ACE2 and TMPRSS2 remain unchanged in the inflamed colon of patients with ulcerative colitis as compared with uninflamed healthy individuals $\mathrm{s}^{26,27,55}$. Another study using bulk RNA sequencing or microarray transcriptomics from tissue samples reported elevated ACE2 levels in patients with ulcerative colitis compared with healthy individuals ${ }^{54}$. ACE2 is increased in active disease and in patients who subsequently require anti-TNF therapy. This study also noted normalization of ACE2 expression in active ulcerative colitis upon anti-cytokine therapy. In addition, a study reported a $70 \%$ increase in colonic ACE2 mRNA expression in patients with ulcerative colitis and a $30 \%$ increase in Crohn's disease ${ }^{56}$. Furthermore, a large study in patients with IBD (Crohn's disease, $n=193$; ulcerative colitis, $n=158$; non-IBD control individuals, $n=51$ ) observed that colonic ACE2 is higher in inflamed colon of patients with Crohn's disease and ulcerative colitis than in healthy individuals ${ }^{57}$. Network analyses highlighted hepatocyte nuclear factor $4 a(\mathrm{HNF} 4 \mathrm{~A})$ as a key regulator of ileal ACE2 levels, whereas pro-inflammatory stimuli, such as cytokines, upregulate ACE2 in colonic organoids from patients with ulcerative colitis. Finally, another study did not observe an effect of both biologic and non-biological IBD medications (aminosalicylates, corticosteroids, thiopurines, TNF blockers) on ACE2 and TMPRSS2 receptor expression in uninflamed intestines ${ }^{58}$. Collectively, heterogeneous findings were obtained in studies on ACE2 mRNA expression levels in IBD, potentially owing to differences in patient cohorts, medical therapies or comorbidities. However, studies on protein levels suggest decreased ACE2 protein levels in intestinal epithelial cells in patients with Crohn's disease during active inflammation ${ }^{27}$, indicating that these patients might be protected from local inflammation in SARS-CoV-2 infection.

Additional data showed upregulation of soluble ACE2, a shedding receptor that might sequester SARSCoV-2 and prevent its cellular entry, in the blood of patients with $\mathrm{IBD}^{59,60}$. Interestingly, increased levels of soluble ACE2 were detected in the sera of patients

Table 1 | Immunosuppressive and biologic therapies in patients with IBD and liver transplant recipients in the context of COVID-19

\begin{tabular}{|c|c|c|c|}
\hline Therapy & Target & $\begin{array}{l}\text { Risk of opportunistic or } \\
\text { viral infections } \mathbf{s}^{73,79,83,138}\end{array}$ & $\begin{array}{l}\text { Evidence for } \\
\text { aggravated COVID-19 } \\
\left(\text { REFS }^{68,85,108,124}\right)\end{array}$ \\
\hline \multicolumn{4}{|l|}{ IBD } \\
\hline High-dose corticosteroids & Multiple & Yes & Yes \\
\hline JAK inhibitors (tofacitinib) & JAK1/3 & Yes & No \\
\hline Anti-TNF agents & TNF & Yes and no (studies vary) & No \\
\hline Vedolizumab & $\alpha 4 / \beta 7$ integrin & No & No \\
\hline Ustekinumab & IL-12/IL-23 & No & No \\
\hline \multicolumn{4}{|l|}{ Liver transplantation } \\
\hline Tacrolimus & Calcineurin & Yes & No \\
\hline Ciclosporin & Calcineurin & Yes & No \\
\hline Mycophenolate mofetil & $\begin{array}{l}\text { Inosin monophosphate } \\
\text { dehydrogenase }\end{array}$ & Yes & $Y_{e s}{ }^{a}$ \\
\hline Everolimus & mTOR & Yes & No \\
\hline
\end{tabular}

IBD, inflammatory bowel disease. aLimited clinical evidence. 
with IBD, which is consistent with the idea that these patients might at least be partially able to prevent cellular infection via soluble ACE2.

\section{COVID-19 and IBD epidemiology}

Initial studies reported a low incidence of COVID-19 in patients with IBD. For instance, in a cohort of 318 patients with IBD in Wuhan, China, no cases of COVID-19 were found ${ }^{61}$, potentially owing to early warnings to patients and preventive actions to protect this potentially vulnerable population. Subsequent studies demonstrated that SARS-CoV-2 infection in patients with IBD is not $\operatorname{rare}^{62}$. A cross-sectional, observational study showed that more than $10 \%$ ( 82 of 805 ) of patients with IBD in a large referral centre up until April 2020 had confirmed or suspected infection ${ }^{63}$. Although approximately $50 \%$ of affected patients reported diarrhoea, flare-ups during SARS-CoV-2 infection were rare $(<2 \%)$ and $27 \%$ of patients temporarily withdrew from their IBD treatment because of COVID-19; $80 \%$ of the patients had mild disease (79.3\%), whereas the presence of dyspnoea was associated with moderate-to-severe infection. Multicentre studies on cohorts of 97 and 87 patients with COVID-19 and IBD suggested that the risk of COVID-19 in patients with IBD is similar to that in the general population $^{64,65}$ and identified age $>65$ years as a risk factor for pneumonia and hospitalization ${ }^{64}$. Consistently, additional studies revealed that children and younger $(<20$ years of age) patients with IBD usually have mild COVID-19 (REF ${ }^{66}$ ). An additional cohort study of 5,302 patients with IBD suggested the presence of obesity as a risk factor for COVID-19 (REF $\left.{ }^{67}\right)$. Another multicentre IBD cohort study of 100 COVID- 19 cases out of 34,763 patients with IBD $(0.29 \%)$ identified the presence of more than one comorbidity (such as diabetes or hypertension) as an independent risk factor for hospitalization and found that all patients who died had comorbidities ${ }^{68}$. Finally, an Italian observational cohort study in 79 patients with IBD and with COVID-19 showed that age $>65$ years, active IBD and comorbidities were significantly associated with COVID-19-related death ${ }^{69}$ and concluded that the prevention of acute IBD flares might avoid fatal COVID-19 outcomes.

\section{Immunosuppressants and biologics in IBD}

A large case series study on COVID-19 in 97 patients with IBD showed that treatment with corticosteroids increased the risk of hospitalization, whereas treatment with monoclonal antibodies was associated with a reduced risk of pneumonia and hospitalization ${ }^{64}$. Moreover, in a cohort study with 39 COVID-19 cases out of 5,302 patients with IBD, the rate of COVID-19 infection was similar between patients treated with immunosuppression $(0.8 \%)$ and those who were not $(0.64 \%)$, indicating that the use of systemic immunosuppression was not associated with an increased risk of COVID-19 (REF. ${ }^{67}$ ). Another study of 82 patients with IBD and COVID-19 showed that immunosuppression was not related to severity of disease ${ }^{63}$. Similar results were obtained in a study of 259 patients with IBD receiving biologic agents (TNF blockers, vedolizumab, ustekinumab $)^{70}$. Although aminosalicylates and combined immunosuppression with thiopurines and TNF blockers had been initially associated with worse outcomes in patients with IBD and COVID-19 (REF. ${ }^{71}$ ), data from the SECURE-IBD registry $(>4,000 \text { cases })^{72}$ confirmed corticosteroids (15\%), rather than other drugs or general immunosuppression, as a key risk factor for intensive care unit treatment, ventilation or death. The anti-integrin antibody vedolizumab ${ }^{73-76}$ was not associated with an increased risk for these endpoints (5\%), while the IL-12 and IL-23 cytokine blocker ustekinumab $^{77-79}(2 \%)$, TNF blockers (2\% versus $4 \%$ without or with methotrexate or thiopurine therapy), and the JAK1 and JAK3 blocker tofacitinib (3\%) had the lowest risks ${ }^{80}$. Additional risk factors for intensive care unit treatment, ventilation or death in IBD were two or more comorbidities and age $>60$ years ${ }^{80}$.

These findings highlight that (with the exception of high-dose corticosteroids in IBD) immunosuppression per se is not associated with an increased risk for severe COVID-19. In fact, some studies in the general population or in vitro experiments indicated a potentially protective effect of immunosuppressive or cytokine blocker therapy in COVID-19. For instance, thiopurine analogues and mycophenolate mofetil synergistically inhibited coronavirus proteases, whereas the calcineurin inhibitor tacrolimus showed antiviral activity for human coronaviruses, at least in vitro ${ }^{81,82}$. Moreover, TNF blockers in particular were suggested to inhibit pro-inflammatory cytokine release and cytokine storms in COVID-19 in adults and children ${ }^{41,83}$ (TABLE 1; FIG. 1). However, large interventional studies are lacking. Additional studies explored the concept that IL-6R blockade via tocilizumab might block cytokine storm syndromes and severe COVID-19 pneumonia ${ }^{84-86}$. However, a prospective randomized trial in 126 patients did not show evidence for efficacy of tocilizumab in COVID-19 pneumonia with fever and elevated C-reactive protein (CRP) levels as compared to standard care ${ }^{84}$. Moreover, in a second trial in 243 patients, IL-6R blockade via tocilizumab was not effective for preventing intubation or death in hospitalized patients with COVID-19 (REF. ${ }^{85}$ ). However, in hospitalized patients with COVID-19 pneumonia, treatment with tocilizumab reduced the need for mechanical ventilation without affecting survival ${ }^{86}$. Finally, the JAK1 and JAK2 inhibitor baricitinib was tested together with remdesivir versus remdesivir alone for the treatment of COVID-19 in hospitalized patients $(n=1,033)$. Combination therapy was more effective at reducing recovery time and improving clinical status in COVID-19 compared with remdesivir monotherapy (28-day mortality was $5.1 \%$ in the combination group versus $7.8 \%$ upon monotherapy ${ }^{87}$, which is consistent with the idea that blockade of cytokine signalling might be effective in the treatment of COVID-19.

In patients with IBD, this idea was supported by several case reports and case series on the use of TNF blockers in adults and children ${ }^{63,67,88,89}$. Moreover, a study with more than 2,000 patients analysed seroconversion in patients with immune-mediated inflammatory diseases (IMIDs; psoriasis, rheumatoid arthritis, spondyloarthritis and IBD) and in healthy individuals as controls. It was 
found that fewer patients with IMIDs receiving cytokine blocker therapy (which do not deplete B cells) had SARS-CoV-2 IgG seroconversion than patients without such therapy and healthy individuals ${ }^{53}$, suggesting that cytokine inhibitors at least partially protect against severe SARS-CoV-2 infection. Conversely, another study ( $n=789$ patients) examined the effects of immunosuppression on COVID-19 in patients with autoimmune and chronic inflammatory diseases ${ }^{90}$. Relative to the control cohort (without autoimmune diseases and immunosuppressive therapy), patients with immunosuppressive

\section{Box $1 \mid$ Recommendations from the ECCO COVID-19 taskforce and the AGA}

\section{ECCO Recommendations ${ }^{95}$}

Should we stop drugs in patients without symptoms suggestive of coronavirus disease 2019 (COVID-19) (not tested or tested negative)?

\section{Do's}

- Do continue immunomodulators.

- Do continue biologics.

- Do continue JAK inhibitors.

- Do reduce corticosteroids whenever possible.

- Do keep infusions in an infusion centre whenever possible.

Don'ts

- Do not reduce the dose of immunomodulators or biologics to prevent severe acute respiratory syndrome coronavirus 2 (SARS-CoV-2) infection.

- Do not switch infliximab to adalimumab in a stable patient, unless it is not possible to provide intravenous infusions.

- Do not assume that patients with inflammatory bowel disease (IBD) are at increased risk of being infected.

Should we stop IBD drugs in patients who are SARS-CoV-2 positive, whether symptomatic or asymptomatic?

Do's

- Do postpone biologics administration.

- Do stop/reduce corticosteroids whenever possible.

- Do stop azathioprine/mercaptopurine therapy.

- Do stop azathioprine in patients in combination therapy with an anti-TNF.

- Do stop JAK inhibitors.

\section{Don'ts}

- Do not continue prednisone at doses above $20 \mathrm{mg}$ per day.

- Do not restart the treatment until a nasopharyngeal swab PCR-SARS-CoV-2 test (if available) indicates a negative result.

\section{AGA Recommendations ${ }^{94}$}

- Patients with IBD who do not have infection with SARS-CoV-2 should not discontinue their IBD therapies and should continue infusion schedules.

- Patients with IBD who have known SARS-CoV-2 but have not developed COVID-19 should hold thiopurines, methotrexate and tofacitinib; dosing of biological therapies should be delayed for 2 weeks of monitoring for symptoms of COVID-19.

- Patients with IBD who develop COVID-19 should hold thiopurines, methotrexate, tofacitinib and biological therapies during the viral illness; these can be restarted after complete symptom resolution or, if available, when follow-up viral testing is negative or serologic tests demonstrate the convalescent stage of illness.

- The severity of COVID-19 and the severity of IBD should result in careful risk-benefit assessments regarding treatments for COVID-19 and escalating treatments for IBD.

AGA, American Gastroenterological Association; ECCO, European Crohn's and Colitis Organisation. ECCO recommendations adapted with permission from REF. ${ }^{95}$, Oxford University Press. AGA recommendations adapted with permission from REF. ${ }^{94}$, Elsevier $(\odot 2020$ by the AGA Institute). therapy in autoimmunity had a markedly reduced risk of severe acute respiratory distress syndrome. Thus, among patients hospitalized with COVID-19, those receiving immunosuppressive treatment for autoimmune diseases appeared to be protected from severe lung failure.

Thus, cytokine blockers might even exert protective roles in COVID-19, possibly owing to suppression of hyperinflammation. Particularly remarkable is the outcome in patients with IBD treated with anti-TNF and anti-IL-12 and anti-IL-23 therapy. Given the potentially pathogenic role of IL-23 and $\mathrm{T}_{\mathrm{H}} 17$ cells in COVID$19\left(\mathrm{REF}^{10}\right)$, this point requires further investigation. In agreement with a potentially pathogenic role of IL-23 in COVID-19, case reports described asymptomatic infections in patients with psoriasis ${ }^{91,92}$ or psoriasis and Crohn's disease ${ }^{93}$ treated with anti-IL-23 (risankizumab) therapy.

In the absence of large interventional clinical trials, current recommendations from the American Gastroenterological Association (AGA) ${ }^{94}$ and the European Crohn's and Colitis Organisation (ECCO) ${ }^{95}$ suggest the maintenance of immunosuppressive and biologic therapy in patients with IBD in the absence of SARS-CoV-2 infection and the postponement (biologic agents) or cessation of (immunosuppressants) therapy in patients with active disease (BOX 1). Importantly, prednisolone therapy should be stopped or reduced, if possible, as corticosteroids rather than other drugs or general immunosuppression might increase the risk of intensive care unit treatment, ventilation or death.

\section{COVID-19 in autoimmune hepatitis}

A small initial study from Italy included ten patients with COVID-19 with autoimmune hepatitis (AIH) in biochemical remission under immunosuppressive therapy $(n=8)$ or in acute onset AIH requiring high-dose corticosteroids $(n=2)^{96}$. Liver enzymes during SARS-CoV-2 infection remained unchanged or even improved upon treatment with corticosteroids. Despite the development of pneumonia in five cases, none of the patients died, suggesting that immunosuppression in AIH does not impair the course of the disease. This observation was supported by a report from Belgium of 85 patients with $\mathrm{AIH}$, in which it was shown that these patients were rarely affected by COVID-19 despite the use of immunosuppressive therapy. Marked adherence of patients with AIH to protective guidelines during the COVID-19 pandemic was noted in this observational study ${ }^{97}$. Clinical symptoms compatible with COVID-19 were noted in seven patients, but only one patient had positive SARS-CoV-2 PCR testing. The uneventful course of disease in AIH supported the notion that immunosuppressive therapy should not be stopped during the pandemic. A third study analysed data from 70 patients with AIH (83\% on immunosuppressive therapy) and 932 patients with chronic liver disease as controls in the context of SARS-CoV-2 infection ${ }^{98}$. Importantly, the study did not observe significant differences between both groups with regard to key outcome parameters, including hospitalization, admission to intensive care units and death. Collectively, these findings indicate that immunosuppressive therapy in $\mathrm{AIH}$ 
should not routinely be discontinued during the course of COVID-19 (REF. ${ }^{98}$ ).

Based on the currently available data, several recommendations for AIH therapy in the context of COVID-19 have been made ${ }^{98-100}$. It has been suggested that hospitalized patients with AIH should receive standard therapy at the usual dose for treatment of acute flares ${ }^{99}$. Moreover, the American Association for the Study of Liver Diseases (AASLD) and the European Association for the Study of the Liver (EASL) recommend that patients with AIH on immunosuppression without COVID-19 should not decrease immunosuppression ${ }^{98-100}$. By contrast, in the presence of COVID-19, lowering of immunosuppression - particularly of anti-metabolites in patients with lymphopenia - should be considered ${ }^{98-100}$. Moreover, EASL suggests that budesonide should be used for therapy in patients with AIH and with COVID-19 to reduce and minimize systemic glucocorticoid exposure for the management of acute flares of AIH. However, in patients with $\mathrm{AIH}$ on immunosuppression, corticosteroid dosing should be maintained at low levels to prevent adrenal insufficiency. Finally, the Asian Pacific Association for the Study of the Liver (APASL) suggests the continuation of immunosuppressive therapy in patients with $\mathrm{AIH}$ with mild COVID-19 infection, while discontinuation of corticosteroid treatment in patients with AIH with severe COVID-19 should be avoided ${ }^{101}$.

\section{COVID-19 in liver transplantation}

Initial data on COVID-19 in solid organ transplant (SOT) recipients receiving immunosuppressive therapy raised concerns that this infection might lead to high mortality ${ }^{102,103}$ (TABLE 1). A study of 36 kidney transplant recipients receiving immunosuppressive therapy demonstrated lower $\mathrm{CD}^{+}$and $\mathrm{CD}^{+} \mathrm{T}$ cell counts in peripheral blood. Furthermore, more-rapid clinical progression of disease was observed compared with the general population ${ }^{102}$, indicating a high early mortality rate among kidney transplant recipients ( $28 \%$ at 3 weeks). A second study of 778 SOT recipients (including 110 liver transplant recipients) in Spain found that the incidence of COVID-19 in SOT recipients was twofold higher than in the general population ${ }^{104} ; 89 \%$ of patients with COVID-19 were admitted to hospital, whereas adjustment of immunosuppression was performed in $85 \%$. Overall, 27\% of SOT recipients diagnosed with COVID-19 died and univariate analysis yielded lung transplantation and hospital-acquired COVID-19 infection as risk factors for death ${ }^{104}$. A third study in 18 transplant recipients (44\% kidney, 33\% liver, 22\% heart) with COVID-19 reported a case-fatality rate of $27 \%{ }^{102,103}$, suggesting that SARS-CoV-2 infection results in a severe course of COVID-19 in many transplant recipients. Similarly, in a study of 11 SOT (kidney and liver) recipients with HIV, there was a high mortality rate of $36 \%{ }^{105}$. By contrast, a study of 21 SOT recipients diagnosed with COVID-19 demonstrated favourable short-term outcomes, with less than $10 \%$ mortality, but highlighted that $50 \%$ required treatment at intensive care units and that those with concomitant infections had more severe illness ${ }^{106}$. Finally, a Spanish observational study in 46 adult SOT recipients with SARS-CoV-2 infection reported a higher mortality in SOT recipients compared with matched controls (37 versus $23 \%)^{107}$. In this study, $72 \%$ of patients underwent transitory discontinuation of immunosuppressants due to potential or confirmed drug-drug interactions. Potential concerns included the possibility of drug-drug interactions between tacrolimus and some of the treatments with antiviral effects such as lopinavir-ritonavir and azithromycin ${ }^{108}$.

Additional studies focused on the effects of COVID-19 in liver transplant recipients. Although it was postulated that immunosuppressed patients with COVID-19 are at increased risk of severe clinical manifestations of disease, preliminary data suggested that COVID-19 in liver transplant recipients has similar or lower incidence rates than the general population ${ }^{109}$. In this context, liver transplant recipients, just like the general population, are at risk of exposure to SARS-CoV-2 infection outside and inside the hospital and nosocomial COVID-19 outbreaks have been reported in liver transplant wards ${ }^{110}$. However, case reports suggested that liver transplant recipients who have had COVID-19 might have prolonged viral shedding due to concomitant immunosuppressive therapy ${ }^{111-113}$.

Several studies in liver transplant recipients with COVID-19 have been conducted; however, it should be noted that most liver transplant studies reported short-term outcomes on uncontrolled case series with partially conflicting results. An international prospective study across Europe on 57 liver transplant recipients with SARS-CoV-2 infection reported that $72 \%$ were hospitalized, 19\% developed acute respiratory distress syndrome and $12 \%$ had a fatal outcome ${ }^{114}$. Remarkably, the majority of the patients who died had a history of cancer. This finding is in agreement with studies from recent meta-analyses showing that patients with COVID-19 and cancer have a higher fatality rate than patients with COVID-19 without cancer ${ }^{15,116}$. In the European study on liver transplant recipients with COVID-19 (REF. ${ }^{114}$ ), immunosuppression was reduced in $22(37 \%)$ recipients and discontinued in $4(7 \%)$ and there was no observed effect of this modulation of immunosuppressive therapy on clinical outcome.

A Spanish multicentre study of 111 liver transplant recipients with COVID- 19 showed that $86 \%$ were admitted to hospital, $20 \%$ required respiratory support and $11 \%$ were treated at intensive care units ${ }^{117}$. Overall, a mortality rate of $18 \%$ was reported. This study found marked effects of concomitant immunosuppression on COVID-19 outcomes. In fact, immunosuppression containing mycophenolate was a highly statistically significant predictor of severe COVID-19, particularly at doses higher than $1,000 \mathrm{mg} /$ day. The reasons for this observation are currently unclear, although a $\mathrm{T}$ cell-depleting effect of this drug has been suggested as a potential explanation ${ }^{117,118}$. By contrast, calcineurin inhibitors or everolimus had no negative effects in liver transplant recipients and complete withdrawal of immunosuppression yielded no benefits. Based on these findings, the researchers suggested that selective mycophenolate dose reduction or withdrawal rather than complete immunosuppression withdrawal could prevent severe COVID-19 in liver transplant recipients. 
Another multicentre cohort study of 151 adult liver transplant recipients with COVID-19 from 18 countries showed that admission to the intensive care unit $(28 \%$ versus $8 \%$ ) and invasive ventilation (20\% versus $5 \%)$ were more frequent in the liver transplant group compared with a control cohort (general population) ${ }^{119}$. By contrast, only $19 \%$ of patients in the former cohort died and higher age, serum creatinine levels and non-liver cancer were risk factors for death among liver transplant recipients. Finally, a study from Europe on 243 adult liver transplant recipients with laboratory-confirmed SARS-CoV-2 infection showed that $84 \%$ required hospitalization and $19 \%$ were treated at intensive care units ${ }^{120}$. Moreover, $20 \%$ of patients with COVID-19 died, mainly owing to respiratory failure. Although age $>70$ years had a negative effect on clinical outcome, tacrolimus use had a positive effect on the survival of liver transplant recipients in COVID-19. The researchers postulated that these data might encourage clinicians to use tacrolimus at stable doses even in cases of SARS-CoV-2 infection.

Several of these studies looked at the effects of immunosuppression on COVID-19 in more detail ${ }^{114,117,120}$. While two studies reported favourable results of calcineurin inhibitor therapy on survival ${ }^{114,120}$, the third study noted that baseline immunosuppression containing tacrolimus caused a trend towards a reduced risk of severe COVID-19 (REF. ${ }^{117}$ ). Thus, these studies underlined the idea that chronic immunosuppression could exert a protective effect against the severe forms of COVID-19, arguing against complete withdrawal of immunosuppression in liver transplant recipients ${ }^{121}$. Unlike calcineurin inhibitors, mycophenolate mofetil might facilitate COVID-19 and should be used cautiously unless further studies become available.

\section{Box 2 | WHO definitions of severe and non-severe COVID-19 forms and} WHO recommendations on the use of corticosteroids in COVID-19

\section{WHO definitions ${ }^{139}$}

Critical coronavirus disease 2019 (COVID-19)

Defined by the criteria for acute respiratory distress syndrome, sepsis, septic shock or other conditions that would normally require the provision of life-sustaining therapies such as mechanical ventilation (invasive or non-invasive) or vasopressor therapy.

\section{Severe COVID-19}

Defined by any of:

- Oxygen saturation $<90 \%$ on room air.

- Respiratory rate $>30$ breaths per minute in adults and children $>5$ years old; 60 or more in children less than 2 months; 50 or more in children $2-11$ months old; and 40 or more in children $1-5$ years old.

- Signs of severe respiratory distress (that is, accessory muscle use, inability to complete full sentences; in children, very severe chest wall indrawing, grunting, central cyanosis, or presence of any other general danger signs).

Non-severe COVID-19

Defined as absence of any signs of severe or critical COVID-19.

\section{WHO Recommendations ${ }^{139}$}

\section{Recommendation 1}

"We recommend systemic corticosteroids rather than no corticosteroids for the treatment of patients with severe and critical COVID-19 (strong recommendation)".

\section{Recommendation 2}

"We suggest not to use systemic corticosteroids in the treatment of patients with non-severe COVID-19 (conditional recommendation)".

Adapted by permission from BMJ Publishing Group Limited (REF. ${ }^{139}$ ).
The currently available studies indicate that long-term liver transplant recipients are at higher risk for severe COVID-19 than short-term liver transplant recipients ${ }^{109}$, suggesting that comorbidities in the former rather than intense immunosuppression in the latter patient population is associated with disease severity and fatal outcomes. Although immunosuppression might be beneficial in suppressing augmented immune responses in the body in COVID-19, only some studies reported protective effects of immunosuppression with tacrolimus ${ }^{120}$. Based on the currently available data, most national and international societies advise against modifications of immunosuppression in mild COVID-19, whereas reduction or discontinuation of antiproliferative and lymphocyte-depleting therapies should be considered in cases with severe lymphopenia, superinfections and progressive pneumonia ${ }^{114,117,120}$. However, it should be considered that a marked reduction or complete withdrawal of immunosuppressive therapy could potentially augment COVID-19-related inflammation, while maintenance of immunosuppressive therapy might impair antiviral immune responses and B cell antibody production. Thus, additional interventional studies are needed to define the right treatment regimen and the ideal time point for adapting immunosuppressive therapy in liver transplant recipients with SARS-CoV-2 infection ${ }^{122}$. Moreover, the potential interactions between experimental COVID-19 treatments and immunosuppressive therapies require further investigation.

\section{Conclusions and future perspectives}

The studies discussed in this Review have provided new insights into the effects of immunosuppression in patients with gastrointestinal diseases and COVID- 19. In patients with IBD, high-dose corticosteroid treatment before SARS-CoV-2 infection has been identified as a key risk factor for aggravated outcomes, suggesting that corticosteroid therapy for IBD in general should be reduced or avoided if possible ${ }^{123-125}$ (BOX 1). By contrast, the WHO generally recommends systemic corticosteroids for the treatment of patients with severe COVID-19 in intensive care units ${ }^{126}$ (BOX 2). The WHO recommendations were based on data indicating that systemic corticosteroids probably reduced the risk of 28-day mortality compared with no corticosteroid therapy ${ }^{127}$. However, in patients with non-severe COVID-19, systemic corticosteroids might increase the risk of 28-day mortality and are thus not currently recommended ${ }^{127,128}$.

Non-glucocorticoid immunosuppression in patients with IBD and liver transplant recipients per se appears not to be a risk factor for aggravated outcomes and could even exert a protective effect against the most severe forms of COVID-19. Therefore, complete withdrawal of immunosuppression is not advisable 33,71,114,117. $^{\text {. }}$ Studies in liver transplant recipients have additionally highlighted the potential benefits of tacrolimus therapy, whereas one study described the potentially deleterious effects of mycophenolate therapy. These results should be considered in attempts to modulate immunosuppression in liver transplant recipients during COVID-19 but they require further confirmatory studies ${ }^{114,117}$. 
Box 3 | Key IOIBD and AASLD recommendations for COVID-19 vaccination in patients with IBD and liver transplant recipients

\section{IOIBD recommendations: patients with IBD ${ }^{137}$}

- Patients with inflammatory bowel disease (IBD) should be vaccinated against severe acute respiratory syndrome coronavirus 2 (SARS-CoV-2); the best time to administer SARS-CoV-2 vaccination in patients with IBD is at the earliest opportunity to do so.

- SARS-CoV-2 vaccines, including messenger RNA vaccines, replication-incompetent vector vaccines, inactivated vaccines and recombinant vaccines, are safe to administer to patients with IBD.

- SARS-CoV-2 vaccination should not be deferred because a patient with IBD is receiving immune-modifying therapies.

- Patients with IBD vaccinated with SARS-CoV-2 should be counselled that vaccine efficacy may be decreased when receiving systemic corticosteroids.

\section{AASLD recommendations: liver transplant recipients ${ }^{136}$}

- Vaccination against SARS-CoV-2 is recommended for all liver transplant recipients.

- The best time to administer the SARS-CoV-2 vaccine in liver transplant recipients is likely at least 3 months post-liver transplant when immunosuppression is lower; however, immunization may be given as early as 6 weeks post-transplant, especially for the highest risk individuals with other comorbid factors.

- A reduction in immunosuppression is not recommended in liver transplant recipients solely to elicit an immune response to immunization against SARS-CoV-2 as there is a risk of acute cellular rejection with lower immunosuppression.

- Avoid SARS-CoV-2 vaccination in liver transplant recipients with active acute rejection, those being treated for acute cellular rejection, or those on high daily doses of corticosteroids until the episode is resolved and their baseline immunosuppression re-established.

- Given the life-saving nature of liver transplantation, deceased donor transplantation should not be delayed in a patient who received a SARS-CoV-2 vaccine.

- If the patient is due for a second dose of vaccine in the immediate post-transplant period, this may be delayed 6 weeks to elicit a better immune response.

- Potential live liver donors and recipients of live donor livers should receive the second dose of the SARS-CoV-2 vaccine at least 2 weeks before transplantation.

AASLD, American Association for the Study of Liver Diseases; COVID-19, coronavirus disease 2019; IOIBD, International Organization for the study of Inflammatory Bowel Diseases. IOIBD recommendations adapted by permission from BMJ Publishing Group Limited (REF. ${ }^{137}$ ). AASLD recommendations adapted with permission from REF. ${ }^{136}$, Wiley. This article is protected by copyright. All rights reserved. gastrointestinal diseases and/or immunosuppression (BOX 3). Currently, there is a constantly growing vaccine pipeline for SARS-CoV-2 and 19 phase I, 23 phase I/II, 6 phase II and 20 phase III studies are in progress $^{129}$. These approaches include vaccines with RNA (Pfizer-BioNTech, Moderna), DNA, proteins or protein subunits (Novavax), viral vectors (Johnson \& Johnson, Oxford-AstraZeneca, Gamaleya Research Institute of Epidemiology and Microbiology), and inactivated vaccines or attenuated viruses (Sinopharm, Sinovac, Sinopharm's unit in Wuhan, Bharat Biotech) to prevent SARS-CoV-2 infection ${ }^{130}$. Moreover, several vaccines have been approved in Europe, the USA and the UK, including two mRNA vaccines (Moderna, Pfizer-BioNTech) and one vaccine with an adenovirus vector in the UK (Oxford-AstraZeneca). In addition, a combined adenoviral vector vaccine, denoted Gam-COVID-Vac (Sputnik V from Gamaleya Research Institute), that is based on rAd type 26 (rAd26) and rAd type 5 (rAd5), was recently approved (emergency permit) in Hungary ${ }^{131}$. Although these vaccines hold great promise for the prevention of SARS-CoV-2 infection in patients on immunosuppressive or biologic therapy, more data on their efficacy are clearly needed as patients with IMIDs have not been studied in detail in the clinical trials so far. In the absence of available data on vaccination effects, antibody responses in these patients should be closely monitored as concomitant immunosuppressive or biologic therapy might affect $\mathrm{T}$ cell immunity and antibody production by B cells. In agreement with this concept, reduced pneumococcal and H1N1 influenza vaccine response rates were previously observed in patients with IBD receiving thiopurines and anti-TNF antibodies ${ }^{132-134}$. Moreover, infliximab treatment in IBD has been associated with attenuated serological antibody responses to SARS-CoV-2 upon infection that were further reduced by concomitant immunosuppressive therapy ${ }^{135}$. Thus, in patients taking immunosuppressive drugs, including biologic agents and small-molecule inhibitors, the key concerns of SARS-CoV-2 vaccines currently relate to the theoretical risk of suboptimal vaccine responses rather than to their adverse effects ${ }^{132}$. Nevertheless, patients with IBD and liver transplant recipients, just like the general population, are at risk of COVID-19 and should be encouraged to follow strict hygiene and social distancing measures and to consider early access to vaccination whenever available ${ }^{136,137}$.

Published online 29 June 2021
In IBD, protective effects of the use of cytokine blockers on COVID-19 have been reported in some studies and require further investigation. Thus, controlled clinical trials and further research on the use of immunosuppressive and biologic agents are of critical relevance for an improved understanding of COVID-19 pathogenesis and its effect on IMIDs in gastrointestinal diseases.

SARS-CoV-2 mRNA vaccines should be considered for the prevention of infection in patients with
1. Cobb, N. L. et al. Comparison of clinical features and outcomes in critically ill patients hospitalized with COVID-19 versus influenza. Ann. Am. Thorac. Soc. 18 632-640 (2021).

2. Bhatraju, P. K. et al. Covid-19 in critically ill patients in the Seattle region - case series. N. Engl. J. Med. 382, 2012-2022 (2020).

3. Wu, F. et al. A new coronavirus associated with human respiratory disease in China. Nature 579, 265-269 (2020).

4. Guan, W. J. et al. Clinical Characteristics of Coronavirus Disease 2019 in China. N. Engl. J. Med. 382

1708-1720 (2020)

5. Wölfel, R. et al. Virological assessment of hospitalized patients with COVID-19. Nature 581, 465-469 (2020).
6. Zhou, J. et al. Infection of bat and human intestinal organoids by SARS-CoV-2. Nat. Med. 26, 1077-1083 (2020).

7. Coronaviridae Study Group of the International Committee on Taxonomy of Viruses. The species severe acute respiratory syndrome-related coronavirus: classifying 2019-nCoV and naming it SARS-CoV-2. Nat. Microbiol. 5, 536-544 (2020).

8. Rehman, S. U., Shafique, L., Ihsan, A. \& Liu, O. Evolutionary Trajectory for the Emergence of Novel Coronavirus SARS-CoV-2. Pathogens 9, 240 (2020).

9. Matsuyama, S. et al. Enhanced isolation of SARS-CoV- 2 by TMPRSS2-expressing cells. Proc. Natl Acad. Sci. USA 117, 7001-7003 (2020)

10. De Biasi, S. et al. Marked T cell activation, senescence, exhaustion and skewing towards $\mathrm{TH} 17$ in patients with COVID-19 pneumonia. Nat. Commun. 11, 3434 (2020).

11. Li, Q. et al. Early transmission dynamics in Wuhan China, of novel coronavirus-infected pneumonia. N. Engl. J. Med. 382, 1199-1207 (2020).

12. Shah, H., Khan, M. S. H., Dhurandhar, N. V. \& Hegde, V. The triumvirate: why hypertension, obesity, and diabetes are risk factors for adverse effects in patients with COVID-19. Acta Diabetol. 58, 831-843 (2021).

13. Lu, R. et al. Genomic characterisation and epidemiology of 2019 novel coronavirus: implications for virus origins and receptor binding. Lancet 395, 565-574 (2020).

14. Reynolds, H. R. et al. Renin-angiotensin-aldosterone system inhibitors and risk of Covid-19. N. Engl. J. Med. 382, 2441-2448 (2020). 
15. Yan, R. et al. Structural basis for the recognition of SARS-CoV-2 by full-length human ACE2. Science 367 1444-1448 (2020).

16. Zhang, H., Penninger, J. M., Li, Y., Zhong, N. \& Slutsky, A. S. Angiotensin-converting enzyme 2 (ACE2) as a SARS-CoV-2 receptor: molecular mechanisms and potential therapeutic target. Intensive Care Med. $\mathbf{4 6}$. 586-590 (2020)

17. Hashimoto, T. et al. ACE2 links amino acid malnutrition to microbial ecology and intestinal inflammation. Nature 487, 477-481 (2012).

18. Byrnes, J. J. et al. Effects of the ACE2 inhibitor GL1001 on acute dextran sodium sulfate-induced colitis in mice. Inflamm. Res. 58, 819-827 (2009).

19. Hoffmann, M. et al. SARS-CoV-2 cell entry depends on ACE2 and TMPRSS2 and is blocked by a clinically proven protease inhibitor. Cell 181, 271-280 (2020)

20. Guo, Y. R. et al. The origin, transmission and clinical therapies on coronavirus disease 2019 (COVID-19) outbreak - an update on the status. Mil. Med. Res. 7 , 11 (2020).

21. Redd, W. D. et al. Prevalence and characteristics of gastrointestinal symptoms in patients with severe acute respiratory syndrome coronavirus 2 infection in the United States: a multicenter cohort study. Gastroenterology 159, 765-767 (2020).

22. Pan, L. et al. Clinical characteristics of COVID-19 patients with digestive symptoms in Hubei, China: a descriptive, cross-sectional, multicenter study. Am. J. Gastroenterol. 115, 766-773 (2020).

23. Dong, Z. Y., Xiang, B. J., Jiang, M., Sun, M. J. \& Dai, C. The prevalence of gastrointestinal symptoms, abnormal liver function, digestive system disease and liver disease in COVID-19 infection: a systematic review and meta-analysis. J. Clin. Gastroenterol. 55 67-76 (2021).

24. Neurath, M. F. COVID-19 and immunomodulation in IBD. Gut 69, 1335-1342 (2020)

25. Grover, S. et al. High prevalence of gastrointestinal manifestations of COVID-19 infection in hospitalized patients with cancer. J. Clin. Gastroenterol. 55, 84-87 (2021).

26. Xiao, F. et al. Evidence for gastrointestinal infection of SARS-CoV-2. Gastroenterology 158, 1831-1833 (2020).

27. Patankar, J. V. et al. The SARS-CoV-2 attachment receptor ACE2 is decreased in Crohn's disease and regulated by microbial and inflammatory signaling. Gastroenterology 160, 925-928 (2021).

28. Lamers, M. M. et al. SARS-CoV-2 productively infects human gut enterocytes. Science 369, 50-54 (2020).

29. Patel, K. P. et al. Gastrointestinal, hepatobiliary, and pancreatic manifestations of COVID-19. J. Clin. Virol. 128, 104386 (2020)

30. Cai, Q. et al. COVID-19: abnormal liver function tests. J. Hepatol 73, 566-574 (2020).

31. Yadlapati, S. et al. Prevailing patterns of liver enzymes in patients with COVID-19 infection and association with clinical outcomes. Ann. Gastroenterol. 34, 224-228 (2021)

32. Huang, C. et al. Clinical features of patients infected with 2019 novel coronavirus in Wuhan, China. Lancet 395, 497-506 (2020)

33. Mehta, P. et al. COVID-19: consider cytokine storm syndromes and immunosuppression. Lancet 395 1033-1034 (2020)

34. Sadeghi, A. et al. Th 17 and Treg cells function in SARS-CoV2 patients compared with healthy controls. J. Cell. Physiol. 236, 2829-2839 (2021).

35. Parrot, T. et al. MAIT cell activation and dynamics associated with COVID-19 disease severity. Sci. Immunol. 5, eabe 1670 (2020)

36. Remy, K. E. et al. Severe immunosuppression and not a cytokine storm characterizes COVID-19 infections. JCl Insight 5, e 140329 (2020)

37. Leppkes, M. et al. Vascular occlusion by neutrophil extracellular traps in COVID-19. EBioMedicine 58, 102925 (2020).

38. Schurink, B. et al. Viral presence and immunopathology in patients with lethal COVID-19: a prospective autopsy cohort study. Lancet Microbe 1 e290-e299 (2020)

39. Lipsitch, M., Grad, Y. H., Sette, A. \& Crotty, S Cross-reactive memory $\mathrm{T}$ cells and herd immunity to SARS-CoV-2. Nat. Rev. Immunol. 20, 709-713 (2020).

40. Chiotos, K. et al. Multicenter interim guidance on use of antivirals for children with coronavirus disease 2019/severe acute respiratory syndrome coronavirus 2. J. Pediatric Infect. Dis. Soc. 10, 34-48 (2021)

41. Harwood, R. et al. A national consensus management pathway for paediatric inflammatory multisystem syndrome temporally associated with COVID-19 (PIMS-TS): results of a national Delphi process. Lancet Child Adolesc. Health 5, 133-141 (2021).

42. Sekine, T. et al. Robust T cell immunity in convalescent individuals with asymptomatic or mild COVID-19. Cell 183, 158-68 (2020)

43. Swadling, L. \& Maini, M. K. T cells in COVID-19 - united in diversity. Nat. Immunol. 21, 1307-1308 (2020)

44. Grifoni, A. et al. Targets of T cell responses to SARS CoV-2 coronavirus in humans with COVID-19 disease and unexposed individuals. Cell 181, 1489-1501 (2020).

45. Zhang, J. Y. et al. Single-cell landscape of immunological responses in patients with COVID-19. Nat. Immunol. 21, 1107-1118 (2020)

46. Peng, $Y$. et al. Broad and strong memory $C D 4^{+}$ and $\mathrm{CD}^{+} \mathrm{T}$ cells induced by SARS-CoV- 2 in UK convalescent individuals following COVID-19. Nat. Immunol. 21, 1336-1345 (2020).

47. Schulien, I. et al. Characterization of pre-existing and induced SARS-CoV-2-specific CD8 ${ }^{+} \mathrm{T}$ cells. Nat. Med. 27, 78-85 (2020)

48. Parackova, Z., Bloomfield, M., Klocperk, A. \& Sediva, A. Neutrophils mediate Th 17 promotion in COVID-19 patients. J. Leukoc. Biol. 109, 73-76 (2021).

49. Pairo-Castineira, E et al. Genetic mechanisms of critical illness in Covid-19. Nature 591, 92-98 (2021)

50. Sahin, U. et al. COVID-19 vaccine BNT162b1 elicits human antibody and TH1 T cell responses. Nature 586, 594-599 (2020).

51. Meckiff, B. J. et al. Imbalance of regulatory and cytotoxic SARS-CoV-2-reactive $\mathrm{CD}^{+}{ }^{+} \mathrm{T}$ cells in COVID-19. Cell 183, 1340-13536 (2020).

52. Pedersen, S. F. \& Ho, Y. C. SARS-CoV-2: a storm is raging. J. Clin. Invest. 130, 2202-2205 (2020).

53. Simon, D et al. Patients with immune-mediated inflammatory diseases receiving cytokine inhibitors have low prevalence of SARS-CoV-2 seroconversion. Nat. Commun. 11, 3774 (2020).

54. Potdar, A. A. et al. Altered intestinal ACE2 levels are associated with inflammation, severe disease, and response to anti-cytokine therapy in inflammatory bowel disease. Gastroenterology 160, 809-822 (2021).

55. Burgueno, J. F. et al. Expression of SARS-CoV-2 entry molecules ACE2 and TMPRSS2 in the gut of patients with IBD. Inflamm. Bowel Dis. 26, 797-808 (2020).

56. Nowak, J. K. et al. Age, inflammation, and disease location are critical determinants of intestinal expression of SARS-CoV-2 receptor ACE2 and TMPRSS2 in inflammatory bowel disease. Gastroenterology 159, 1151-1154 (2020).

57. Verstockt, B. et al. Intestinal receptor of SARS-CoV-2 in inflamed IBD tissue seems downregulated by HNF4A in ileum and upregulated by interferon regulating factors in colon. J. Crohns Colitis 15, 485-498 (2021)

58. Suarez-Farinas, $M$. et al. Intestinal inflammation modulates the expression of ACE2 and TMPRSS2 and potentially overlaps with the pathogenesis of SARS-CoV-2-related disease. Gastroenterology 160 287-301 (2021).

59. Batlle, D. Wysocki, J \& Satchell, K Soluble angiotensin-converting enzyme 2 : a potential approach for coronavirus infection therapy? Clin. Sci. 134, 543-545 (2020)

60. Garg, M. et al. Imbalance of the renin-angiotensin system may contribute to inflammation and fibrosis in IBD: a novel therapeutic target? Gut 69, 841-851 (2019).

61. An, P. et al. Protection of 318 inflammatory bowel disease patients from the outbreak and rapid spread of COVID-19 infection in Wuhan, China. Lancet Gastroenterol. Hepatol. 5, 525-527 (2020).

62. Berte, R. et al. Seroprevalence of SARS-CoV2 in IBD patients treated with biological therapy. J. Crohns Colitis 15, 864-868 (2021).

63. Guerra, I. et al. Incidence, clinical characteristics, and evolution of SARS-CoV-2 infection in patients with inflammatory bowel disease: a single-center study in Madrid, Spain. Inflamm. Bowel Dis. 27, 25-33 (2021)

64. Allocca, M. et al. Patients with inflammatory bowel disease are not at increased risk of COVID-19: a large multinational cohort study. J. Clin. Med. 9, 3533 (2020).

65. Khan, N. et al. Are patients with inflammatory bowel disease at an increased risk of developing SARS-CoV-2 than patients without inflammatory bowel disease? Results from a nationwide Veterans' Affairs Cohort Study. Am. J. Gastroenterol. 116, 808-810 (2021).

66. Brenner, E. J. et al. Benign evolution of SARS-Cov2 infections in children with inflammatory bowel disease: results from two international databases. Clin Gastroenterol Hepatol 19, 394-396 (2020).

67. Burke, K. E. et al. Immunosuppressive therapy and risk of COVID-19 infection in patients with inflammatory bowel diseases. Inflamm. Bowel Dis. 27 155-161 (2020).

68. Derikx, L. et al. Clinical outcomes of Covid-19 in patients with inflammatory bowel disease: a nationwide cohort study. J. Crohns Colitis 15 529-539 (2021)

69. Bezzio, C. et al. Outcomes of COVID-19 in 79 patients with IBD in Italy: an IG-IBD study. Gut 69 1213-1217 (2020).

70. Bossa, F. et al. Impact of the COVID-19 outbreak and the serum prevalence of SARS-CoV-2 antibodies in patients with inflammatory bowel disease treated with biologic drugs. Dig. Liver Dis. 53, 277-282 (2021)

71. Ungaro, R. C. et al. Effect of IBD medications on COVID-19 outcomes: results from an international registry. Gut 70, 725-732 (2021).

72. Brenner, E. J., Ungaro, R. C., Colombel, J. F. \& Kappelman, M. D. SECURE-IBD Database Public Data Update. covidibd.org (2 May 2021).

73. Feagan, B. G. et al. Vedolizumab as induction and maintenance therapy for ulcerative colitis. N. Engl. J. Med. 369, 699-710 (2013).

74. Sandborn, W. J. et al Vedolizumab as induction and maintenance therapy for Crohn's disease. N. Engl. J. Med. 369, 711-721 (2013)

75. Zundler, S., Becker, E., Schulze, L. L. \& Neurath, M. F Immune cell trafficking and retention in inflammatory bowel disease: mechanistic insights and therapeutic advances. Gut 68, 1688-1700 (2019).

76. Fischer, A. et al. Differential effects of $\alpha 4 \beta 7$ and GPR 15 on homing of effector and regulatory $T$ cells from patients with UC to the inflamed gut in vivo. Gut 65, 1642-1664 (2016).

77. Sandborn, W. J. et al. Ustekinumab induction and maintenance therapy in refractory Crohn's disease. N. Engl. J. Med. 367, 1519-1528 (2012).

78. Sands, B. E. et al. Ustekinumab as induction and maintenance therapy for ulcerative colitis. $N$. Engl. J. Med. 381, 1201-1214 (2019).

79. Neurath, M. F. Targeting immune cell circuits and trafficking in inflammatory bowel disease. Nat. Immunol. 20, 970-979 (2019).

80. Agrawal, $\mathrm{M}$. et al. Characteristics and outcomes of IBD patients with COVID-19 on tofacitinib therapy in the SECURE-IBD Registry. Inflamm. Bowel Dis. 27, 585-589 (2021)

81. Cheng, K. W. et al. Thiopurine analogs and mycophenolic acid synergistically inhibit the papainlike protease of Middle East respiratory syndrome coronavirus. Antivir. Res. 115, 9-16 (2015).

82. Carbajo-Lozoya, J. et al. Human coronavirus NL63 replication is cyclophilin A-dependent and inhibited by non-immunosuppressive cyclosporine A-derivatives including Alisporivir. Virus Res. 184, 44-53 (2014).

83. Feldmann, M. et al. Trials of anti-tumour necrosis factor therapy for COVID-19 are urgently needed. Lancet 395, 1407-1409 (2020).

84. Salvarani, C. et al. Effect of tocilizumab vs standard care on clinical worsening in patients hospitalized with COVID-19 pneumonia: a randomized clinical trial. JAMA Intern. Med. 181, 24-31 (2021).

85. Stone, J. H. et al. Efficacy of tocilizumab in patients hospitalized with Covid-19. N. Engl. J. Med. 383, 2333-2344 (2020).

86. Salama, C. et al. Tocilizumab in patients hospitalized with Covid-19 pneumonia. N. Engl. J. Med. 384, 20-30 (2021)

87. Kalil, A C et al. Baricitinib plus remdesivir for hospitalized adults with Covid-19. N. Engl. J. Med. 384, 795-807 (2021).

88. Dolinger, M. T. et al. Pediatric Crohn disease and multisystem inflammatory syndrome in childre (MIS-C) and COVID-19 treated with infliximab. J. Ped. Gastroenterol. Nutr. 71, 153-155 (2020).

89. Abdullah, A., Neurath, M. F. \& Atreya, R. Mild COVID-19 symptoms in an infliximab-treated ulcerative colitis patient: can ongoing anti-TNF therapy protect against the viral hyperinflammatory response and avoid aggravated outcomes? Visc. Med. 36, 338-342 (2020).

90. Monreal, E. et al. The impact of immunosuppression and autoimmune disease on severe outcomes in patients hospitalized with COVID-19. J. Clin. Immunol. 41, 315-323 (2020)

91. Ward, M. \& Gooderham, M. Asymptomatic SARS $\mathrm{CoV} 2$ infection in a patient receiving risankizumab, an inhibitor of interleukin 23. JAAD Case Rep. 7, 60-61 (2021). 
92. Wang, C. J. \& Truong, A. K. COVID-19 infection on IL-23 inhibition. Dermatol Ther 33 e13893 (2020)

93. Messina, F. \& Piaserico, S. SARS-CoV-2 infection in a psoriatic patient treated with IL-23 inhibitor. J. Eur. Acad. Dermatol. Venereol. 34, e254-e255 (2020).

94. Rubin, D. T., Feuerstein, J. D., Wang, A. Y. \& Cohen, R. D. AGA clinical practice update on management of inflammatory bowel disease during the COVID-19 pandemic: expert commentary. Gastroenterology 159, 350-357 (2020).

95. Magro, F. et al. Inflammatory bowel disease management during the COVID-19 outbreak: the ten do's and don'ts from the ECCO-COVID Taskforce. J. Crohns Colitis 14, S798-S806 (2020).

96. Gerussi, A. et al. Coronavirus disease 2019 (COVID-19) in autoimmune hepatitis: a lesson from immunosuppressed patients. Hepatol. Commun. $\mathbf{4}$ 1257-1262 (2020)

97. Verhelst, X., Somers, N., Geerts, A., Degroote, H. $\S$ Van Vlierberghe, $H$. Health status of patients with autoimmune hepatitis is not affected by the SARSCoV-2 outbreak in Flanders, Belgium. J. Hepatol. 74 240-241 (2021).

98. Marjot, T. et al. SARS-CoV-2 infection in patients with autoimmune hepatitis. J. Hepatol. 74, 1335-1343 (2021).

99. Lleo, A., Invernizzi, P., Lohse, A. W., Aghemo, A $\&$ Carbone, M. Management of patients with autoimmune liver disease during COVID-19 pandemic. J. Hepatol. 73, 453-455 (2020).

100. Mohammed, A., Paranji, N., Chen, P. H. \& Niu, B. COVID-19 in chronic liver disease and liver transplantation: a clinical review. J. Clin. Gastroenterol. 55, 187-194 (2021).

101. Force, A. C.-T., Lau, G. \& Sharma, M. Clinical practice guidance for hepatology and liver transplant providers during the COVID-19 pandemic: APASL expert panel consensus recommendations. Hepatol. Int. 14 415-428 (2020).

102. Akalin, E. et al. Covid-19 and kidney transplantation. N. Engl. J. Med. 382, 2475-2477 (2020).

103. Fernandez-Ruiz, M. et al. COVID-19 in solid organ transplant recipients: a single-center case series from Spain. Am. J. Transplant. 20, 1849-1858 (2020).

104. Coll, E. et al. COVID-19 in transplant recipients: the Spanish experience. Am. J. Transplant 21. 1825-1837 (2021).

105. Mehta, S. A. et al. Incidence and outcomes of COVID19 in kidney and liver transplant recipients with HIV: report from the National HOPE in Action Consortium. Transplantation 105, 216-224 (2021).

106. Yi, S. G. et al. Early experience with COVID-19 and solid organ transplantation at a US high-volume transplant center. Transplantation 104, 2208-2214 (2020).

107. Miarons, M. et al. COVID-19 in solid organ transplantation: a matched retrospective cohort study and evaluation of immunosuppression management. Transplantation 105, 138-150 (2020).

108. Forns, X. \& Navasa, M. Liver transplant immunosuppression during the covid-19 pandemic. Gastroenterol. Hepatol. 43, 457-463 (2020).

109. Di Maira, T. \& Berenguer, M. COVID-19 and liver transplantation. Nat. Rev. Gastroenterol. Hepatol. 17, 526-528 (2020)

110. Alconchel, F. et al. Severe COVID-19 after liver transplantation, surviving the pitfalls of learning on-the-go: three case reports. World J. Hepatol. 12 870-879 (2020)
111. Dhand, A. et al. Successful liver transplantation in a patient recovered from COVID-19. Transpl. Infect. Dis. 23, e13492 (2021)

112. Niess, H. et al. Liver transplantation in a patient after COVID-19 - rapid loss of antibodies and prolonged viral RNA shedding. Am. J. Transplant. 21 1629-1632 (2020).

113. Wei, L., Liu, B., Zhao, Y. \& Chen, Z. Prolonged shedding of SARS-CoV-2 in an elderly liver transplant patient infected by COVID-19: a case report. Ann. Palliat. Med. https://doi.org/10.21037/apm-20-996 (2020).

114. Becchetti, C. et al. COVID-19 in an international European liver transplant recipient cohort. Gut 69, 1832-1840 (2020).

115. Zhang, L. et al. Clinical characteristics of COVID-19infected cancer patients: a retrospective case study in three hospitals within Wuhan, China. Ann. Oncol. 31, 894-901 (2020)

116. Nahshon, C. et al. Outcomes of diagnosed COVID-19 cancer patients: concerning results of a systematic review. J. Chemother. https://doi.org/10.1080/112000 9X.2021.1899442 (2021).

117. Colmenero, J. et al. Epidemiological pattern, incidence and outcomes of COVID-19 in liver transplant patients. J. Hepatol. 74, 148-155 (2020).

118. Allison, A. C. \& Eugui, E. M. Mechanisms of action of mycophenolate mofetil in preventing acute and chronic allograft rejection. Transplantation $\mathbf{8 0}$ (Suppl. 2), 181-190 (2005)

119. Webb, G. J. et al. Outcomes following SARS-CoV-2 infection in liver transplant recipients: an international registry study. Lancet Gastroenterol. Hepatol. 5, 1008-1016 (2020)

120. Belli, L. S. et al. Protective role of tacrolimus, deleterious role of age and comorbidities in liver transplant recipients with Covid-19: results from the ELITA/ELTR multi-center European study. Gastroenterology 160, 1151-1163 (2021).

121. Rodriguez-Peralvarez, M., Salcedo, M., Colmenero, J. $\&$ Pons, J. A. Modulating immunosuppression in liver transplant patients with COVID-19. Gut 70 1412-1414 (2021).

122. Azzi, Y., Bartash, R., Scalea, J., Loarte-Campos, P. \& Akalin, E. COVID-19 and solid organ transplantation: a review article. Transplantation 105, 37-55 (2021).

123. Lukin, D. J. et al. Baseline disease activity and steroid therapy stratify risk of COVID-19 in patients with inflammatory bowel disease. Gastroenterology 159 1541-1544 (2020).

124. Brenner, E. J. et al. Corticosteroids, but not TNF antagonists, are associated with adverse COVID-19 outcomes in patients with inflammatory bowel diseases: results from an international registry. Gastroenterology 159, 481-491 (2020).

125. Schett, G., Sticherling, M. \& Neurath, M. F. COVID-19 risk for cytokine targeting in chronic inflammatory diseases? Nat. Rev. Immunol. 20, 271-272 (2020)

126. Horby, P. et al. Dexamethasone in hospitalized patients with Covid-19 - preliminary report. N. Engl. J. Med. 384, 693-704 (2021)

127. Cheng, W. et al. Efficacy and safety of corticosteroid treatment in patients with COVID-19: a systematic review and meta-analysis. Front. Pharmacol. 11, 571156 (2020).

128. Shuto, $\mathrm{H}$. et al. A systematic review of corticosteroid treatment for noncritically ill patients with COVID-19. Sci. Rep. 10, 20935 (2020).
129. Parker, E. P. K., Shrotri, M. \& Kampmann, B. Keeping track of the SARS-CoV-2 vaccine pipeline. Nat. Rev. Immunol. 20, 650 (2020).

130. Mahase, E. Covid-19: Novavax vaccine efficacy is $86 \%$ against UK variant and $60 \%$ against South African variant. BMJ 372, n296 (2021).

131. Logunov, D. Y. et al. Safety and efficacy of an rAd26 and $\mathrm{rAd} 5$ vector-based heterologous prime-boost COVID-19 vaccine: an interim analysis of a randomised controlled phase 3 trial in Russia. Lancet 397, 671-681 (2021).

132. Alexander, J. L. et al. SARS-CoV-2 vaccination for patients with inflammatory bowel disease: a British Society of Gastroenterology Inflammatory Bowel Disease section and IBD Clinical Research Group position statement. Lancet Gastroenterol. Hepatol. 6 218-224 (2021)

133. Fiorino, G. et al. Effects of immunosuppression on immune response to pneumococcal vaccine in inflammatory bowel disease: a prospective study. Inflamm. Bowel Dis. 18, 1042-1047 (2012).

134. Cullen, G., Bader, C., Korzenik, J. R. \& Sands, B. E. Serological response to the $2009 \mathrm{H} 1 \mathrm{~N} 1$ influenza vaccination in patients with inflammatory bowel disease. Gut 61, 385-391 (2012).

135. Kennedy, N. A. et al. Anti-SARS-CoV-2 antibody responses are attenuated in patients with IBD treated with infliximab. Gut 70, 865-875 (2021).

136. Fix, O. K. et al. AASLD Expert Panel consensus statement: vaccines to prevent COVID-19 infection in patients with liver disease. Hepatology https://doi.org 10.1002/hep.31751 (2021).

137. Siegel, C. A. et al. SARS-CoV-2 vaccination for patients with inflammatory bowel diseases: recommendations from an international consensus meeting. Gut 70 635-640 (2021)

138. Sandborn, W. J. et al. A randomized tria of ustekinumab, a human interleukin-12/23 monoclonal antibody, in patients with moderateto-severe Crohn's disease. Gastroenterology 135 1130-1141 (2008)

139. Rochwerg, B. et al. A living WHO guideline on drugs for covid-19. BMJ 370, m3379 (2020).

\section{Acknowledgements}

M.F.N. is supported by grants DFG SFB 643; SFB 1181 . TRR241; FOR2438; KFO257; BMBF DZI ilmmune.

\section{Competing interests}

M.F.N. has served as an advisor for Abbvie, Pentax, Giuliani, MSD, Takeda, Roche and Boehringer.

\section{Peer review information}

Nature Reviews Gastroenterology \& Hepatology thanks M. Berenguer, M. Kappelman, who co-reviewed with E. Brenner, and the other, anonymous, reviewer(s) for their contribution to the peer review of this work.

Publisher's note

Springer Nature remains neutral with regard to jurisdictional claims in published maps and institutional affiliations.

RELATED LINKS

Johns Hopkins University of Medicine. Coronavirus

Resource Center: https://coronavirus.jhu.edu/map.htn

(C) Springer Nature Limited 2021 